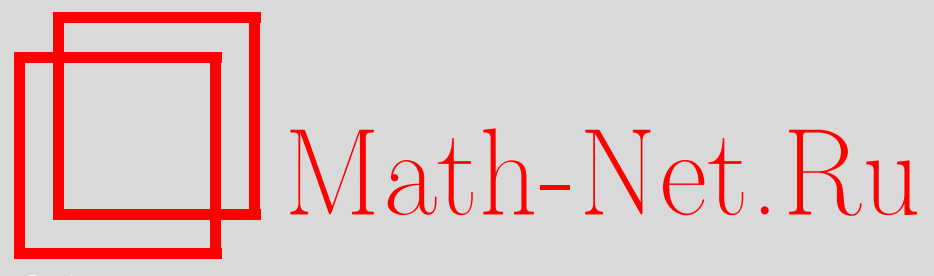

С. М. Натанзон, А. М. Пратусевич, Пространство модулей горенштейновых квазиоднородных особенностей поверхностей, УМН, 2011, том 66, выпуск 5, 189-190

DOI: https://doi.org/10.4213/rm9445

Использование Общероссийского математического портала Math-Net.Ru подразумевает, что вы прочитали и согласны с пользовательским соглашением http://www.mathnet.ru/rus/agreement

Параметры загрузки:

IP : 18.207 .199 .55

26 апреля 2023 г., 16:51:02

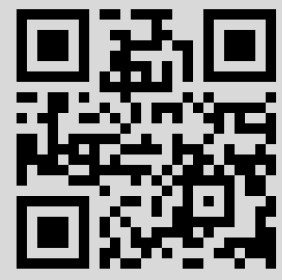




\title{
Пространство модулей горенштейновых квазиоднородных особенностей поверхностей
}

\author{
С. М. Натанзон, А. М. Пратусевич
}

1. В этой работе мы описываем топологию пространства горенштейновых гиперболических квазиоднородных особенностей поверхностей. Мы определяем количество компонент связности этого пространства и описываем их топологию. Квазиоднородные особенности поверхностей и их аффинные координатные кольца активно изучались в 70-е годы И. В. Долгачевым, Дж. Милнором, В. Нойманом, Г. Пинкхэмом и другими. Назовем особенность гиперболической, если соответствующий орбифолд гиперболический, т. е. имеет вид $\mathbb{H} / \Gamma$, где $\mathbb{H}-$ гиперболическая плоскость и $Г$ - фуксова группа. Нормальная изолированная особенность поверхности горенштейнова, если на проколотой окрестности особой точки существует везде ненулевая голоморфная 2-форма. Например, все изолированные особенности полных пересечений горенштейновы. И. В. Долгачев [1] показал, что горенштейновы гиперболические квазиоднородные особенности поверхностей уровня $m$ однозначно соответствуют особым $m$-коспинорным расслоениям над компактными гиперболическими римановыми орбифолдами, т. е. линейным расслоениям, $m$-я степень которых изоморфна касательному расслоению.

Имеется взаимно однозначное соответствие между $m$-коспинорными и $m$-спинорными расслоениями. Классические (т. е. $m=2$ ) спинорные расслоения на компактных римановых поверхностях совпадают с тэта-характеристиками Римана. Их современная интерпретация и классификация предложены М. Ф. Атьей [2] и Д. Мамфордом [3]. Классификация 2-спинорных расслоений и их модулей на некомпактных поверхностях дана в [4]. Описание пространств модулей $m$-спинорных расслоений дано в [5] для компактных поверхностей с проколами и в [6], [7] для поверхностей с произвольной конечно порожденной фундаментальной группой.

2. ОПРЕДЕЛЕНИЕ. Пусть $p$ - точка компактного гиперболического риманова орбифолда $P$. Пусть $\pi_{1}^{0}(P, p) \subset \pi_{1}(P, p)$ - подмножество фундаментальной группы орбифолда, состоящее из элементов, представимых простыми контурами. Отображение $\sigma: \pi_{1}^{0}(P, p) \rightarrow \mathbb{Z} / m \mathbb{Z}$ назовем $m$-функцией $А р \oint a$, если для любых элементов $a, b \in \pi_{1}^{0}(P, p)$ выполнены условия: 1) $\left.\sigma\left(b a b^{-1}\right)=\sigma(a) ; 2\right) \sigma\left(a^{-1}\right)=-\sigma(a)$, если порядок элемента $a$ не равен 2 ; 3$) \sigma(a b)=\sigma(a)+\sigma(b)$, если $a$ и $b$ можно представить простыми контурами таким образом, чтобы точка $p$ была их единственной общей точкой и их индекс пересечения не 0 ; 4) $\sigma(a b)=\sigma(a)+\sigma(b)-1$, если: а) индекс пересечения $a$ и $b$ равен 0 , б) $a$ и $b$ можно представить простыми контурами таким образом, чтобы точка $p$ была их единственной общей точкой, в) ориентированные контуры $a, b$ и $(a b)^{-1}$ свободно гомотопны попарно не пересекающимся простым контурам $\tilde{a}, \tilde{b}, \tilde{c}$, ориентации которых противоположны индуцированным комплексной структурой сферы с 3 дырами, которую они отсекают от $P$; 5 ) для всякого эллиптического элемента $c$ порядка $p$ число $p \sigma(c)+1$ делится на $m$.

Теорема 1. Существует естественное взаимно однозначное соответствие между горенштейновыми гиперболическими квазиоднородными особенностями поверхностей уровня $m$ и т-функциями Арфа на компактных гиперболических римановых орбиболдах.

Доказательство основано на связи между $m$-спинорными расслоениями на орбифолде $\mathbb{H} / \Gamma$ и поднятиями фуксовой группы $\Gamma \subset P S L(2, \mathbb{R})$ на единственное $m$-листное накрытие $\varphi: G_{m} \rightarrow \operatorname{PSL}(2, \mathbb{R})$.

Работа выполнена при поддержке грантов РФФИ-10-01-00678-a, НШ-8462.2010.1, SFB 611 (DFG), RCMM (Liverpool). 
3. Опишем теперь простейшие топологические инварианты $m$-функций Арфа $\sigma$. $\mathrm{K}$ ним мы отнесем род $g$ орбифолда $P$ и порядки $p_{1}, \ldots, p_{r}$ особых точек на $P$. Из определения $m$-функций Арфа сразу следует, что группа Mod гомотопических классов сохраняющих ориентацию автогомеоморфизмов орбифолда $P$ естественно действует на множестве $\Sigma$ всех $m$-функций Арфа на $P$.

Теорема 2. Множество $\Sigma$ всех т-функций Арфа на компактном гиперболическом орбифолде $P$ сигнатуры $\left(g: p_{1}, \ldots, p_{r}\right)$ не пусто, тогда и только тогда, когда порядки $p_{1}, \ldots, p_{r}$ взаимно просты с $m$ и $и\left(p_{1} \cdots p_{r}\right)\left(\sum_{1 \leqslant i \leqslant r} \frac{1}{p_{i}}-(2 g-2)-r\right)$ делится на т. В этом случае число т-функиий Арфа равно $\mathrm{m}^{2 g}$. Действие группъ Мод на непустом множестве $\Sigma$ транзитивно, если $g=0$ или же если $g>1$ и но; имеет ровно две орбиты, если $g>1$ и т четно; имеет число орбит, равное числу делителей НОД $\left(m, p_{1}-1, \ldots, p_{r}-1\right)$, если $g=1$.

Доказательство использует явный вид дэновских образующих группы Mod.

4. Используем теперь $m$-функции Арфа для изучения пространства $S^{m}$ гиперболических горенштейновых квазиоднородных особенностей поверхностей уровня $m$. Пространство $S^{m}$ естественно распадается на подмножества $S_{g: p_{1}, \ldots, p_{r}}^{m}$, отвечающие простейшим топологическим инвариантам соответствующих $m$-функций Арфа. Из теоремы 2 сразу следует

Теорема 3. Пространство $S_{g: p_{1}, \ldots, p_{r}}^{m}$ не пусто, тогда и толъко тогда, когда порлдки $p_{1}, \ldots, p_{r}$ взаимно просты с т $и\left(p_{1} \cdots p_{r}\right)\left(\sum_{1 \leqslant i \leqslant r} \frac{1}{p_{i}}-(2 g-2)-r\right)$ делится на $\mathrm{m}$. Непустое пространство $S_{g: p_{1}, \ldots, p_{r}}^{m}$ свлзно, если $g=0$ или же если $g>1$ и нечетно; имеет ровно две компоненты связности, если $g>1$ и т четно; имеет число компонент свлзности, равное числу делителей НОД $\left(m, p_{1}-1, \ldots, p_{r}-1\right)$, если $g=1$.

Из теорем 1-3 и теоремы Фрике-Клейна [8] в форме [9], выводится

ТЕОРема 4. Каждая компонента связности пространства $S_{g: p_{1}, \ldots, p_{r}}^{m}$ гомеоморфна $\mathbb{R}^{d} / G$, где $d=6 g-6+2 r u G \subset \operatorname{Mod}-$ дискретнал группа.

\section{Список литературы}

[1] I. V. Dolgachev, Math. Ann., 265:4 (1983), 529-540. [2] M. F. Atiyah, Ann. Sci. École Norm. Sup. (4), 4 (1971), 47-62. [3] D. Mumford, Ann. Sci. École Norm. Sup. (4), 4 (1971), 181-192. [4] С. М. Натанзон, Матем. заметки, 45:4 (1989), 111-116. [5] T. J. Jarvis, Internat. J. Math., 11:5 (2000), 637-663. [6] С. Натанзон, А. Пратусевич, УMH, 60:2(362) (2005), 169-170. [7] S. Natanzon, A. Pratoussevitch, J. Lie Theory, 19:1 (2009), 107-148. [8] R. Fricke, F. Klein, Vorlesungen über die Theorie der automorphen Funktionen. B. I, II, Johnson Reprint Corp., New York; Teubner, Stuttgart, 1965. [9] С. М. Натанзон, Модули римановых поверхностей, вещественных алгебраических кривых и их супераналоги, МЦНМО, М., 2003.

C. M. Натанзон (S. M. Natanzon)

ВШЭ; ИФХБ им. А. Н. Белозерского, МГУ; ИТЭФ

E-mail: natanzons@mail.ru

А. М. Пратусевич (А. M. Pratusevich)

University of Liverpool

E-mail: annap@liv.ac.uk
Представлено С. М. Гусейн-Заде Принято редколлегией 09.09.2011 